\title{
Applicability Analysis of Bidding Strategy in Electricity Market
}

\author{
Suyan Zhou ${ }^{1,}$, Fei Chen ${ }^{2}$, Yahui Qiao ${ }^{3}$, Wenzhe Zhang ${ }^{4}$, Kaifeng Zhang ${ }^{1}$, Kun Yuan ${ }^{1}$, Xuemei Dai ${ }^{1}$ \\ ${ }^{1}$ Key Laboratory of Measurement and Control of Complex Systems of Engineering, Southeast University, Nanjing, 210096, PR China \\ ${ }^{2}$ Shanxi Electric Power Company, Xi'an, 710082, PR China \\ ${ }^{3}$ Hohai University, Nanjing, 210098, PR China \\ ${ }^{4}$ Chongqing Electric Power Company, Chongqing, 404100, PR China
}

\begin{abstract}
With the development of the electricity market, competition has been introduced in the generation side. It is the overall development trend of the electricity market reformulation to optimize the allocation of different resources through bidding. Therefore, it is significant to research the bidding strategies of the generation companies and the large consumers. This paper reviews the existing research methods of bidding strategy. According to the different market mechanisms, the market participators will choose different bidding strategies based on their own cases. We analyze the applicability of bidding strategies under the different conditions, and give suggestions on how to select bidding strategy for the different market participators under different conditions.
\end{abstract}

\section{Introduction}

With the development of the electricity market, competition has been introduced in the generation side and retail side. It is the overall development trend of the electricity market reformulation to optimize the allocation of different resources through bidding. Different from the planned power generation under monopoly mode, power generation companies need to achieve their own power generation and profit through bidding in the electricity market.

According to the theory of the economics, generation companies must quote the marginal cost of the unit if they want to maximize their expected profits in a perfectly competitive market. And in this case the market will have the highest operating efficiency [1]. However, the real electricity market is closely to oligopolistic market. This is mainly due to the fact that there are usually only a few dominant firms producing power in an area. In oligopolistic markets, generation companies try to maximize their expected profits by bidding strategically instead of bidding on marginal cost. In this case, the bidding strategies adopted by the generation companies in the market have a direct impact on their profitability.

With the thorough reform of the electric power system in China, the direct power-purchase for large consumers is one of the key points of this reform. So it is significant to research the bidding strategy of the generation companies and the large consumers in the electricity market.

Strategic bidding in the electricity market has been studied by many researchers using the different methods. A remarkable review is presented in [2], in which several different research methods on generation companies' bidding strategy before the year of 2000 are summarized. In [3], some new bidding strategies based on evolutionary game theory are presented.

Previous reviews on bidding strategies in the electricity market have mostly embarked from the perspective of optimal strategy, discussing how to construct the optimal strategy for generation companies. So the most common method based on power generation cost analysis has been often ignored. On the other hand, with the development of the electricity market, some new problems and approaches emerge, which have not been summarized in previous study works. Also the applicability of various bidding strategies under different market conditions are not discussed in previous reviews.

To summarize, the main contributions of this paper are as follows:

To sort and classify the existing research methods on bidding strategies in the electricity market from a more comprehensive perspective. It means that the classification is not from the perspective of how to construct optimal strategy but includes the common method based on power generation cost analysis in practice.

To analyze the applicability of various bidding strategies under the different market conditions and give some suggestions on how to select bidding strategy for the different market participators according to the different market conditions.

The rest of the paper is constructed as follows. In Section II , the existing methods of studying bidding strategy in the electricity market are summarized. In Section III, we analyze the applicability of bidding strategy under the different conditions and give suggestions on how to select bidding strategy for the different market participators under different conditions. 
Conclusions and expectations are presented in Section IV.

\section{The research methods of bidding strategy in electricity market}

Most previous studies on bidding strategy in the electricity market have mostly embarked from the perspective of optimal strategy, discussing how to construct the optimal strategy for generation companies. The most common method based on power generation cost analysis has been ignored. However, it is a practical approach in real market that is easily grasped for most generation companies. In some cases, this method may be sufficient and more effective for generation companies. So in order to understand the research methods of bidding strategy more comprehensively, we sort out the common research methods and compare their advantages and disadvantages.

The methods used to construct bidding strategies include 5 types: method based on power generation cost analysis, method based on market clearing price forecasting, method based on guess of opponents' bidding strategies, method based on game theory and method based on evolutionary game theory. The following describes each of these.

\subsection{The method based on power generation cost analysis}

It is the most fundamental method of constructing bidding strategy for the generation companies $[5,6]$. In some cases, this method may be sufficient and more effective for generation companies than other methods. The principle of the method is simple and it is easily grasped for most generation companies. However, it is hard for generation companies to maximize their own expected profit by this method because the influence of other opponents' bidding strategies are not considered [4].

\subsection{The method based on market clearing price forecasting}

In the electricity market with unified clearing mechanism, this method is commonly used. Such method need to predict the market clearing price based on historical data. If the market price can be accurately estimated and the price is higher than the cost, the generation companies only need to bid slightly lower than the predicted price.

In fact, it is difficult to predict the market clearing price accurately. This is mainly because the accurate prediction need the sufficient historical data and reasonable forecast model. But for the developing electricity market, the historical data is not enough due to the market structure and rules are still being adjusted. Although in a relatively mature market, the accuracy of electricity price forecasting is difficult to guarantee because of the speculation in the market. But it does not mean that prediction is unnecessary. With the development of electricity market, the prediction is still an important reference when generation companies bid. At present, there are many methods to forecast the market price [7 14], such as time series prediction method [7], gray system prediction method, regression analysis [8], neural network [8 12], combination forecast method, wavelet analysis method [12] and so on.

\subsection{The method based on guess of opponents' bidding strategies}

In this method, the opponents' bidding strategies are estimated by probability or fuzzy method. On this basis, the optimal bidding model is established to seek the optimal bidding strategy.

In order to estimate the bidding behavior of the opponent accurately, it is necessary to know the historical bidding data of the competitors. On this basis, the statistical method [15] is used to estimate the bidding behavior of competitors. However, because a lot of bidding data in the market is confidential and the behaviors of the market participants are speculative, it is difficult to grasp the bidding laws of the competitors.

Most of the studies assume that the bidding laws of the competitors are known, such as the assumption of rival offer obeying the discrete probability distribution $[16,17]$, uniform distribution [18] and normal distribution [19 21,23,26,27], or regarding it as a fuzzy set $[22,24]$.

\subsection{The method based on game theory}

The research methods based on classic game theory are mainly divided into the following two categories. The first type is based on the matrix game model. This model is very difficult to apply in practice because it needs to know all the other competitors' strategies. In fact, it is hard to realize. And when the number of the market members is high, the game matrix will be very large and the solution is complicated. Therefore, this method can only analyze small systems and is far from practical applications.

The second type is based on the oligopoly game model, which mainly includes Cournot model, Stackelberg model and supply function model [31 33]. Many simplified assumptions are made in the application of these models, and the equilibrium point obtained in this case may not have much significance for the construction of bidding strategies. Generally, the methods based on the classic game theory are more suitable for rough analysis of the market participants' bidding behaviors.

\subsection{The method based on evolutionary game theory}

Compared with the method based on classic game theory, the evolutionary game theory seeks the equilibrium point through continuous learning in the environment [3]. 
Based on the intelligent agent technology, each market participant is modeled as an independent agent. Each agent knows only its personal information and the public information of the market. But it does not know the private information of other agents. This assumption conforms to the incomplete information game in the real market. At present, this method is widely used in power market simulation research [36 38].

\section{The applicability of bidding strategy in electricity market}

The operation modes of electricity markets in distinct countries are different, and the bidding rules and clearing rules adopted by different markets are also different. According to the different market mechanisms and management, market participators will choose different bidding strategies based on their own cases. In this section, we analyze the applicability of bidding strategy under the different conditions, and give suggestions on how to select bidding strategy for the different market participators under different conditions.

\subsection{The bidding strategies in different electricity market}

According to the time division, the electricity market system can be divided into the medium and long-term contract market, day-ahead market and real-time market. The generation companies can participant in different markets at the same time. Considering the generation companies' objectives in these markets, the bidding strategies adopted in different markets need to be adjusted.

For example, in order to avoid the risk of price fluctuations in the spot market, the goal of most power generation companies is to gain as much power as possible in the medium and long-term contract market.

Therefore, the generation companies usually bid low to improve the probability of bid winning. In this case, it is more appropriate for them to bid based on power generation cost in the medium and long-term contract market. This is mainly because the method based on cost analysis has less risk and makes generation companies win the bid more easily than other methods.

The purpose of most power companies participating in the spot market is to obtain additional economic benefits. Therefore, for these generation companies, they try to achieve the maximum expected profit in the spot market. But if the generation companies bid only based on their own cost, without considering the impact of the opponent's bidding in the spot market, it is not conductive to achieve the goal of maximizing profits. Obviously, it is more beneficial for some generation companies which are familiar with the competitors' information and have sufficient historical data in the spot market. These generation companies can estimate the opponents' bidding behaviors or predict the market clearing price based on these historical data for their reference. However, when the generation companies use these bidding strategies to gain high profits, they will also face different risks, such as market price risk, power generation risk and so on.

\subsection{The bidding strategies of the generation companies with different scales}

In terms of the generation company's scale and market share in the electricity market, it will compete as a pricetaker or a price-maker. The greater the generation company's market share, the greater the ability to influence the market. In the oligopoly market, such large-scale generation companies can indirectly determine the market price [18]. In this case, some small and medium generation companies with high cost become price-taker due to lack of competitiveness in the market. So the generation companies with different scales should take the different bidding strategies to get more profits and enhance competitiveness.

Under uniform clearing pricing rule, the market price is mainly determined by a few large-scale generation companies in this area. So for the small and medium companies, they can bid based on cost to improve the probability of winning the bid. Once the companies win the bid and the marginal clearing price is higher than their bid, such companies will be able to obtain additional profits. In this case, it is wiser to choose the bidding strategy based on cost.

On the contrary, in order to achieve the maximum expected profits, the large-scale power companies, as the price-maker, need to consider the bidding behaviors of a few main competitors who have almost the same strength with them. For such companies, if they can collect more historical data about the market and other competitors, it is more beneficial to bid based on forecast price and estimated competitors' bidding strategies.

\subsection{Different types of generation companies' bidding strategies}

Most of the researches on generation companies' bidding strategies in the market have been carried out around thermal power plants. However, the characteristics of hydropower plants are different from the thermal power plants. This leads to different bidding strategies between them.

The studies on hydropower plants' bidding strategies are mostly from a price-taker perspective $[38,39]$. For the thermal power plant, the most common bidding strategy is to bid based on the fuel cost, which is modeled as a quadratic function of its output power. And fixed cost elements are ignored. On the contrary, the hydropower plants hardly consume fuel after they are completed. The cost of hydropower plants is mainly fixed cost and fuel cost can be neglected. Therefore, the original biding strategy based on cost needs to be adjusted when applied to the hydropower plants.

The bidding strategy based on clearing price forecasting is also commonly used in the electricity market. However, it is difficult to guarantee the accuracy of prediction due to the speculation or collusion in the 
market. This situation is extremely risky for hydropower plants with water constraints. Because the forecast value is slightly higher than the actual market price in a certain period, the hydropower plant will lose the chance of generating electricity. As a result, it is difficult to meet the constraints of water plan $[40,41]$. As a result, it is likely to abandon water in the future because of the limited regulation and storage capacity of the reservoir. And this will cause huge losses. But only by lowering the price to ensure the electricity is obviously uneconomical. Therefore, these original bidding strategies are difficult to meet the hydropower plants' demands and need to be adjusted according to practical experience. The hydropower plants' bidding strategies need for further study and improve.

\subsection{The bidding strategies in the direct power- purchase for large consumers}

With the thorough reform of the electric power system in China, the direct power-purchase for large consumers is one of the key points of this reform. So it is significant to research the bidding strategies of the generation companies and the large consumers in the electricity market.

In this section, we mainly discuss the generation companies' and larger consumers' bidding strategies under the different market clearing mechanisms. Under uniform clearing pricing rule, large-scale generation companies can adjust the bidding strategy according to their own situation and the market conditions. In this rule, the small and medium generation companies can bid based on fuel cost to improve the probability of winning the bid.

Different from uniform pricing, in high-low matching pricing rule, each generation company is paid at the average price of generation company's bid and matched large consumer's bid. It is difficult for generation companies to form a coalition in the electricity market adopting high-low matching clearing mechanism.

Under the circumstance of supply exceeding demand in electricity market, the small and medium generation companies can bid low to ensure a certain proportion of electricity or the maximum bidding electricity cleared. For these companies, bidding based on cost is a more effective and less risky strategy. But for some large companies, they have the ability to collect more historical data about the market and other competitors. It is more beneficial for them to bid based on forecast price and estimated competitors' bidding strategies.

However, large consumers must ensure the bided volume of electricity in order to meet the production demands. So they pay more attention to the bided volume of electricity rather than price in most cases. But the market clearing price is certainly the lower the better for large consumers. So in the electricity market some large consumers will bid the tariff for most of electricity to ensure the bided volume to satisfy production needs. And the offer of small amount of electricity should be as low as possible. This strategy can make large consumers sacrifice the minimum amount of electricity to obtain the lowest clearing price.

\section{Conclusions}

There are many factors that influence the choice of bidding strategies, including bidding rules, market clearing mechanism, and so on. The market participants should choose the suitable strategy according to the market environment and their own situation. At present, there have been many research results of bidding strategies for generation companies, but there are few researches on bidding strategies for large consumers. The bidding strategy for large consumers is worthy of further study.

\section{Acknowledgement}

This work was supported by State Grid Science Project (DZN17201500036).

\section{References}

1. Gans J, King S, Stonecash R, et al. Principles of economics[M]. Cengage Learning, 2011.

2. Wen F, David A K. Bidding strategies in electricity markets[J]. Automation of Electric Power Systems, 2000, 24(14): 1-6.

3. Wang Shuai. A review of bidding strategies for generation companies[J]. Moderm Economic Information, 2009 (23): 107-110.

4. Wang Xifan, Wang Xiuli, Chen Haoyong. Power market theory[M]. Xi'an Jiaotong University Press, 2003.

5. Li Tao, Jiang Chuanwen, Hou Zhijian. A cost offer model on the generation side of power market[J]. Information Journal Hydroelectric Energy, 2001, 19(3): 62-63.

6. Zhang Shaohua, Li Yuzeng. Probabilistic forecasting of short-run marginal cost of power generation under electricity market[J]. Proceedings of the CSEE, 2000, 20(10): 19-22.

7. Nogales F J, Contreras J, Conejo A J, et al. Forecasting next-day electricity prices by time series models[J]. IEEE Transactions on power systems, 2002, 17(2): 342-348.

8. Huang Rixing, Kang Chongqing, Xia Qing. System marginal price forecasting in electricity market[J]. Automation of Electric Power Systems, 2000, 24(22): 9-12.

9. Li Caihua, Wang Zhiwei. Short-term marginal price forecasting with hybrid model[J]. Automation of Electric Power Systems, 2000, 24(22): 9-12.

10. Liu Zhengguo, Gao Fuying. Forecasting marketclearing price in Zhejiang generation market using neural network $[\mathrm{J}]$. Automation of Electric Power Systems, 2002, 26(9): 49-52. 
11. Yang Li, Qiu Jiaju, Jiang Daozhuo. BPN based dayahead unconstrained market clearing price forecasting model[J]. Automation of Electric Power Systems, 2001, 25(19): 11-14.

12. Song chao. Application of wavelet analysis and neural network in electricity price forecasting[D]. Zhejiang University, 2002.

13. Conejo A J, Nogales F J, Arroyo J M. Price-taker bidding strategy under price uncertainty[J]. IEEE Transactions on Power Systems, 2002, 17(4): 10811088.

14. Ni E, Luh P B, Rourke S. Optimal integrated generation bidding and scheduling with risk management under a deregulated power market[J]. IEEE Transactions on Power Systems, 2004, 19(1): 600-609.

15. Bialek W, Callan C G, Strong S P. Field theories for learning probability distributions[J]. Physical review letters, 1996, 77(23): 4693.

16. Zhang D, Wang Y, Luh P B. Optimization based bidding strategies in the deregulated market[C]//Power Industry Computer Applications, 1999. PICA'99. Proceedings of the 21st 1999 IEEE International Conference. IEEE, 1999: 63-68.

17. Song H, Liu C C, Lawarree J. Decision making of an electricity supplier's bid in a spot market[C]//Power Engineering Society Summer Meeting, 1999. IEEE. IEEE, 1999, 2: 692-696.

18. David A K. Competitive bidding in electricity supply[C]//EE Proceedings C-Generation, Transmission and Distribution. IET, 1993, 140(5): 421-426.

19. Wen F, David A K. Optimal bidding strategies and modeling of imperfect information among competitive generators $[\mathrm{J}]$. IEEE transactions on power systems, 2001, 16(1): 15-21.

20. Wen F, David A K. Coordination of bidding strategies in day-ahead energy and spinning reserve markets[J]. International Journal of Electrical Power \& Energy Systems, 2002, 24(4): 251-261.

21. Ma Li, Wen Fushuan. A preliminary investigation on bidding strategies employing step-wise bidding rules $[\mathrm{J}]$. Automation of Electric Power Systems, 2002, 26(9): 16-19.

22. Widjaja M, Sugianto L F, Morrison R E. Fuzzy model of generator bidding system in competitive electricity markets[C]//Fuzzy Systems, 2001. The 10th IEEE International Conference on. IEEE, 2001, 3: 1396-1399.

23. Lamont J W, Rajan S. Strategic bidding in an energy brokerage[J]. IEEE Transactions on Power Systems, 1997, 12(4): 1729-1733.

24. Yang Li, Wen Fushuan. A possibility theory based approach for building optimal bidding strategies in electricity markets[J]. Automation of Electric Power Systems, 2002, 26(23): 12-17.

25. Wen F, David A K. Strategic bidding in reserve market[C]//Advances in Power System Control,
Operation and Management, 2000. APSCOM-00. 2000 International Conference on. IET, 2000, 1: 8085.

26. Wen F, David A K. Optimal bidding strategies for competitive generators and large consumers $[\mathrm{J}]$. International Journal of Electrical Power \& Energy Systems, 2001, 23(1): 37-43.

27. Hao S. A study of basic bidding strategy in clearing pricing auctions $[\mathrm{C}] / /$ Power Industry Computer Applications, 1999. PICA'99. Proceedings of the 21st 1999 IEEE International Conference. IEEE, 1999: 55-60.

28. Diao Qinhua, Lin Jikeng, NI Yixin, et al. Game theory and its applications in power markets[J]. Automation of Electric Power Systems, 2001, 25(1): 19-23.

29. Wu Zhiyong, Kang Chongqing, Xia Qing, et al.Strategic bidding with application of game theory $[\mathrm{J}]$. Automation of Electric Power Systems, 2002, 26(9): 7-11.

30. Contreras J, Candiles O, de la Fuente J I, et al. A cobweb bidding model for competitive electricity markets[J]. IEEE Transactions on Power Systems, 2002, 17(1): 148-153.

31. Song Yiqun, Ni Yixin, Hou Zhijian, et al. A novel model of Gencos strategic behaviors based on conjectured supply function equilibrium[J]. Automation of Electric Power Systems, 2003, 27(13): 15-18

32. Chattopadhyay D. Multicommodity spatial Cournot model for generator bidding analysis[J]. IEEE Transactions on power systems, 2004, 19(1): 267 275.

33. Xian W, Yuzeng L, Shaohua Z. Oligopolistic equilibrium analysis for electricity markets: a nonlinear complementarity approach[J]. IEEE Transactions on Power Systems, 2004, 19(3): 13481355.

34. Xie Shiyu. Evolutionary game theory under bounded rationality $[\mathrm{J}]$. Journal of Shanghai University of Finance and Economics, 2001, 3(5): 39.

35. Gao Jie, Sheng Zhaohan. Elementary groping for evolutionary game theory and its application in electricity market[J]. Automation of Electric Power Systems, 2003, 27(18): 18-21.

36. Fujii Y, Okamura T, Inagaki K, et al. Basic analysis of the pricing processes in modeled electricity markets with multi-agent simulation[C]//Electric Utility Deregulation, Restructuring and Power Technologies, 2004.(DRPT 2004). Proceedings of the 2004 IEEE International Conference on. IEEE, 2004, 1: 257-261.

37. Xiong G, Hashiyama T, Okuma S. An electricity supplier bidding strategy through QLearning[C]//Power Engineering Society Summer Meeting, 2002 IEEE. IEEE, 2002, 3: 1516-1521. 
38. Wang Yeping, Yang Yan, Jing Zhaoxia, et al. Application of intelligent Agent-based simulation to electricity market[J]. Journal of South China University of Technology(Natural Science Edition), 2010, 38(3).

39. Chen Zhixu, Zhang Lizi, Shu Jun, et al. Bidding strategy research for price-taker in electricity market[J]. Electric Power, 2007, 40(6): 1-5.

40. He Li. Research on Bidding Problems for Hydropower Plant in Electricity Market[D]. Huazhong University of Science \& Technology, 2007

41. Shi Xiaojun. Bidding Strategy Research for Hydropower Plant in Electric Power Market[D]. Xi'an University of Technology, 2005. 